\title{
The Pattern of Use of Oral NSAIDs with or without Co- prescription of Gastroprotective Agent for Arthritic Knee by Korean Practitioners
}

\author{
Hee-Chun Kim, $\mathrm{MD}^{1}$, Myung Chul Lee, $\mathrm{MD}^{2}$, Young-Wan Moon, $\mathrm{MD}^{3}$, Seung Suk Seo, $\mathrm{MD}^{4}$, \\ Kwang Won Lee, $\mathrm{MD}^{5}$, Ju Hong Lee, $\mathrm{MD}^{6}$ and Choong-Hyeok Choi, $\mathrm{MD}^{7}$ \\ Department of Orthopedic Surgery, ${ }^{1} \mathrm{CHA}$ Bundang Medical Center, CHA University, Seongnam; ${ }^{2}$ Seoul National University College of Medicine; ${ }^{3}$ Sungkyunkwan \\ University School of Medicine; ${ }^{4}$ Inje University College of Medicine; ${ }^{5}$ Eulji University College of Medicine, Seoul; ${ }^{6}$ Chonbuk National University College of Medicine, \\ Jeonju; ${ }^{7}$ Hanyang University College of Medicine, Seoul, Korea
}

Purpose: The aim of this study was to describe the patterns of use of non-steroidal anti-inflammatory drugs (NSAIDs) for arthritic knees in clinical practice, particularly focusing on the co-prescription of gastroprotective agents for patients with risk factors for adverse gastrointestinal (GI) events. Materials and Methods: Each cross-sectional cohort was a group of outpatients visiting 111 physicians who had prescribed NSAIDs for the patients' arthritic knees for more than three consecutive months. A self-administered questionnaire was completed by each patient and physician.

Results: Nine hundred and forty five patients (48\%) of the whole 1,960 patients belonged to the group with a high or very high risk for NSAIDinduced gastropathy determined by northern California Health Maintenance Organization guidelines. Overall, only less than half of the patients were given co-prescription of gastroprotective agents, regardless of the presence or absence of GI symptoms and irrespective of the level of risk for NSAIDinduced gastropathy.

Conclusions: The physician prescribing NSAIDs for arthritic knees should monitor any GI symptoms and the patient monitor anylevel for NSAIDinduced gastropathy, and be willing to add gastroprotective agents as necessary in order to prevent serious adverse GI events.

Key words: Knee, Arthritis, Non-steroidal anti-inflammatory drugs, Gastroprotective agents, Co-prescription.

\section{Introduction}

Risk factors for development of gastrointestinal (GI) toxicity in patients receiving non-steroidal anti-inflammatory drugs (NSAIDs) for arthritis or other musculoskeletal diseases include $^{1-5)}$ prior history of complicated or uncomplicated

Received October 27, 2011; Revised November 1, 2011;

Accepted November 2, 2011.

Correspondence to: Myung Chul Lee, MD.

Department of Orthopedic Surgery, Seoul National University College of Medicine, 101 Daehak-Ro, Jongno-Gu, Seoul 110-711, Korea.

Tel: +82-2-2072-2367, Fax: +82-2-2072-2718

Email: leemc@snu.ac.kr

This is an Open Access article distributed under the terms of the Creative Common Attribution Non-Commercial License (http://creativecommons.org/licenses/by-nc/3.0/) which permits unrestricted non-commercial use, distribution, and reproduction in any medium, provided the original work is properly cited.

Copyright ๑ 2011. KOREAN KNEE SOCIETY

www.jksrr.org ulcers; increased age; multiple NSAID use; high NSAID dose; concomitant use of corticosteroids or anticoagulants including acetylsalicylic acid ${ }^{6}$; concomitant Helicobactor pylori (H. pylori) infection ${ }^{7,8)}$; comorbidities, such as significant cardiovascular disease; female gender9; and severe rheumatoid arthritis.

Most global and domestic guidelines for clinical practitioners recommend the use of either a cyclooxygenase-2 (COX-2) selective agent or a nonselective NSAID with co-prescription of gastroprotective agents in patients with the risk factors ${ }^{3-5}$.

The aim of this study was to describe the patterns of use of NSAIDs for arthritic knees in clinical practice in Korea, particularly focusing on the co-prescription of gastroprotective agents for patients with or without the risk factors for GI toxicity or adverse GI events.

\section{Materials and Methods}

A survey was performed in the ambulatory of 82 hospitals (62 university and 20 general hospitals) where 111 physicians, members of the Korean Knee Society, had prescribed NSAIDs to 


\section{Kim et al. Oral NSAIDs with or without Co-prescription of Gastroprotective Agent}

relieve symptoms and signs of arthritic knees for more than three consecutive months. This cross-sectional observational study was performed on a single day between August and October 2009, although the exact date of study was different among the survey sites.

All the patients were $\geq 20$ years old, had been taking oral NSAIDs for more than three consecutive months, and required additional prescription of NSAIDs for symptomatic relief of their arthritic knees. A self-administered questionnaire was completed by each of the 2,000 patients who fulfilled those inclusion criteria and by the physicians to get the information of the recent and current prescriptions. The questionnaire for the patient included questions on the risk factors for GI adverse effects, such as age, gender, general condition, any history of peptic ulcer with or without hemorrhage or perforation, concomitant use of anticoagulants or corticosteroids, concomitant $H$. pylori infection, and comorbidities, such as significant cardiovascular disease. It also included questions about the status of the affected knee joint and any adverse GI symptoms. The data from the questionnaires filled up by the physicians were analyzed to investigate the prescribing habits of NSAIDs and gastroprotective agents and to determine whether the physicians took any GI symptoms and the patient's own risk level into consideration when they prescribed medicine.

The patients were stratified according to the risk of developing adverse GI events by using the Standardized Calculator of Risk for Events (SCORE) tool. The SCORE had been developed at Stanford University ${ }^{1)}$ and become the base of the treatment guidelines for the use of NSAIDs that was disseminated by northern California Health Maintenance Organization (HMO). The SCORE tool assigned points for six patient factors including

Table 1. The Prevalence of Risk Factors for Gastrointestinal (GI) Toxicity $(\mathrm{n}=1,960)$

\begin{tabular}{lc}
\hline \multicolumn{1}{c}{ Risk factors } & No. of cases (\%) \\
\hline Age $\geq 65$ years & $1,095(56)$ \\
Prior history GI adverse events & $784(40)$ \\
Comorbidities & $490(25)$ \\
Concomitant use of corticosteroids & $275(14)$ \\
Rheumatoid arthritis & $197(10)$ \\
Concomitant use of aspirin & $117(6)$ \\
Concomitant use of anticoagulant & $39(2)$ \\
Prior history of admission for serious GI adverse & $39(2)$ \\
events & \\
Poor general condition & $20(1)$ \\
\hline
\end{tabular}

Percentage total more than $100 \%$ because of concurrent risk factors. age, gender, morbidity, GI problems, and rheumatoid. Whereas the HMO classification categorized patients as level 1 or lowest risk (1-15 points), level 2 or intermediate risk (16-20 points), and level 3 risk ( 21 points or greater) ${ }^{9}$ we classified the patients into low risk (1-10 points), moderate risk (11-15 points), high risk (16-20 points), and very high risk (21 points or greater) of developing serious GI complications.

\section{Results}

Of the 2,000 patients who completed the questionnaire, 1,960 met the eligibility criteria based on the rules for inclusion and exclusion. Fifty-six per cent of the subjects were more than 65 years of age and $76 \%$ were female.

Table 1 presents the prevalence of individual risk factor for GI complications. One hundred and sixty patients (8\%) were at very high GI risk, and 785 patients (40\%) were considered at high risk for adverse GI events (Table 2).

Among the patients in a high or very high risk group, 321 patients $(34 \%)$ had a prescription of COX-2 inhibitors, 331 patients (35\%) nonselective NSAIDs without co-prescription of gastroprotective agents, and 293 patients (31\%) nonselective NSAIDs plus gastroprotective agents (Table 3). This means, among 542 high or very high-risk patients taking NSAIDs without the co-prescription of gastroprotective agents, 331 patients $(61 \%)$ were given non-selective NSAIDs instead

Table 2. Patients Stratified by Risk of Developing Gastrointestinal Complications using SCORE Tool $(n=1,960)$

\begin{tabular}{lc}
\hline Risk level (level determined by HMO guideline ${ }^{9)}$ ) & No. of cases (\%) \\
\hline Very high $>21$ points (level 3) & $160(8)$ \\
High 16-20 points (level 2) & $785(40)$ \\
Moderate 11-15 points (level 1) & $784(40)$ \\
Low $<11$ points (level 1) & $231(12)$ \\
\hline
\end{tabular}

SCORE: standardized calculator of risk for events, HMO: health maintenance organization.

Table 3. NSAID Prescribed in Patients with High or Very High Risk for GI Toxicity $(\mathrm{n}=945)$

\begin{tabular}{lc}
\hline \multicolumn{1}{c}{ NSAIDs prescribed } & No. of cases (\%) \\
\hline Coxibs without gastroprotective agents & $211(22)$ \\
Coxibs with gastroprotective agents & $110(12)$ \\
Non-selective NSAIDs without gastroprotective agents & $331(35)$ \\
Non-selective NSAIDs with gastroprotective agents & $293(31)$ \\
\hline
\end{tabular}

NSAID: non-steroidal anti-inflammatory drug, GI: gastrointestinal, Coxibs: cyclooxygenase-2 selective NSAIDs. 
Table 4. Utilization of Coxibs in Patients with or without GI Risks or Symptoms

\begin{tabular}{|c|c|}
\hline Patients taking NSAIDs & Coxibs \\
\hline High or very high risk group $(\mathrm{n}=945)$ & $321(34)$ \\
\hline Moderate or low risk group $(\mathrm{n}=1,015)$ & $162(16)$ \\
\hline With GI symptoms ( $\mathrm{n}=941)$ & $223(24)$ \\
\hline No GI symptoms $(\mathrm{n}=1,019)$ & $260(26)$ \\
\hline \multicolumn{2}{|c|}{$\begin{array}{l}\text { Values are presented as number (\%). } \\
\text { NSAID: non-steroidal anti-inflammatory } \\
\text { Coxibs: cyclooxygenase-2 selective NSAIDs. }\end{array}$} \\
\hline \multicolumn{2}{|c|}{$\begin{array}{l}\text { Table 5. Prevalence of Use of Gastroprotective Agents in Patients Taking } \\
\text { Non-steroidal Anti-inflammatory Drugs (NSAIDs) }\end{array}$} \\
\hline Patients taking NSAIDs & Gastroprotection \\
\hline High or very high risk group $(\mathrm{n}=945)$ & $406(43)$ \\
\hline Moderate or low risk group $(\mathrm{n}=1,015)$ & $399(39)$ \\
\hline With GI symptoms ( $\mathrm{n}=941)$ & $412(44)$ \\
\hline No GI symptoms $(\mathrm{n}=1,019)$ & $393(39)$ \\
\hline
\end{tabular}

Values are presented as number (\%).

of selective NSAIDs. Whether the patients had adverse GI symptoms or not did not affect the proportion of patients taking selective NSAIDs (Table 4).

Overall, a gastroprotective therapy was performed in 805 (41\%) patients and only less than half of the patients were given coprescription of gastroprotective agents regardless of the presence or absence of GI symptoms and irrespective of the level of risk for NSAID-induced gastropathy (Table 5). Among the patients using the preventive drugs, 255 (32\%) patients received rebamipide whereas histamine ${ }_{2}\left(\mathrm{H}_{2}\right)$-receptor antagonists $\left(\mathrm{H}_{2} \mathrm{RA}\right)$ were coprescribed for 191 (24\%) patients (Table 6).

\section{Discussion}

The most frequent and significant adverse effect associated with NSAIDs is GI toxicity. The symptoms of GI toxicity include both annoying maladies, such as dyspepsia or disgust, and serious events, such as GI ulcers with hemorrhage or perforation.

Despite all global and domestic guidelines recommending the use of either a COX-2 inhibitor or a nonselective NSAID with co-prescription of gastroprotective agents for patients who are at risk of developing GI toxicity, almost thirty-five per cent of the patients at high or very high risk took nonselective NSAIDs without co-prescription of gastroprotective agents in this study. Besides, more than half of the patients complaining of GI symptoms were not given co-prescription of gastroprotective
Table 6. Types of Gastroprotective Therapy $(n=805)$

\begin{tabular}{lc}
\hline Gastroprotective agents & No. of patients (\%) \\
\hline Cytoprotectant & $347(43)$ \\
Rebamipide & $255(32)$ \\
Ecabet sodium granules & $24(3)$ \\
$\mathrm{H}_{2} \mathrm{RA}$ & $191(24)$ \\
Rranitidine & $105(13)$ \\
Cimetidine & $23(3)$ \\
Gastroprokinetics & $107(13)$ \\
Levosulpride & $27(3)$ \\
Mosapride & $25(3)$ \\
Itopride & $24(3)$ \\
Domperidone & $23(3)$ \\
PPI & $71(9)$ \\
Pantoprazole & $42(5)$ \\
Others & $268(33)$ \\
\hline
\end{tabular}

Percentage total more than $100 \%$ because of concomitant use.

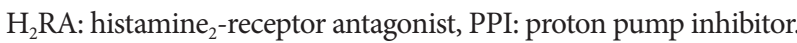

agents, and only a quarter of the patients complaining of GI symptoms were given prescription of selective COX-2 inhibitors in this study.

Overall, only less than half of the patients were given coprescription of gastroprotective agents regardless of the presence or absence of GI symptoms and irrespective of the level of risk for NSAID-induced gastropathy. There have been disparities between medication guidelines and government's reimbursement policies which may modify the enthusiasm of some practitioners for gastroprotection.

With regard to gastroprotective agents, proton pump inhibitor (PPI) or misoprostol has been widely recognized as the most effective one ${ }^{10)}$. PPIs including omeprazole, lansoprazole ${ }^{11)}$, and esomeprazole ${ }^{12)}$ significantly reduced symptomatic ulcers among patients receiving NSAIDs. However, one must also consider potential complications of the long-term PPI use including acceleration of corpus atrophy and, possibly, its role in hip fractures, pneumonia, and pseudomembranous colitis ${ }^{8)}$. It has been shown that the incidence of peptic ulcers associated with NSAID use can be reduced by cotherapy with the synthetic prostaglandin misoprostol. However, poor tolerability is a major limitation of the drug. In a clinical trial ${ }^{13)}, 27.5 \%$ of misoprostoltreated patients withdrew prematurely from the study due to adverse events, most of which were GI complaints including abdominal pain and diarrhea.

Although most global and domestic guidelines recommend the use of PPI or misoprostol for gastroprotection ${ }^{3-5)}$, other 
options that might reduce the risk for NSAID-related upper GI complications are also available. Symptomatic low risk patients without evidence of blood loss may switch to another NSAID (or coxib) or receive treatment with antacids or $\mathrm{H}_{2} \mathrm{RA}^{10)}$.

Selective COX-2 inhibitors (coxibs) are safer to the GI tract than traditional NSAIDs. However, current prevention strategies for patients who need NSAIDs should also take into account the presence of cardiovascular (CV) risk factors, as coxibs and probably post traditional NSAIDs increase the incidence of serious $\mathrm{CV}$ events ${ }^{2}$. Moreover, the introduction of coxibs has not completely eliminated GI adverse events. There have been several reports that COX-2-inhibitor users did not have a reduced risk of a GI bleed compared with patients using nonselective NSAID ${ }^{14)}$. Currently, it is accepted that the choice of an NSAID should be determined by the need to balance each patient's GI and CV risks. Several trials with less potent COX-1 inhibitors showed significant reductions in endoscopic gastric ulcers and erosions $^{10,15,16)}$. The development of COX-2-selective inhibitors and the formation of other new, safer inhibitors should broaden the range of options.

Although acid inhibitors may relieve symptoms, they have not been proven to reduce GI complications, Among $\mathrm{H}_{2} \mathrm{RA}$, only high dose of famotidine has been recognized as a gastroprotective agent in most official guidelines. Recent improvements in the understanding of NSAID-induced damage and new gastroprotective agent development ${ }^{17)}$ would provide an opportunity for effective anti-inflammatory with reduced GI complications.

In summary, strategies for reducing the risk for NSAID-related GI complications remain underused even in high-risk patients and patients with GI symptoms. Thus, the physician prescribing NSAIDS for arthritic knees should determine the level of patient's own risk for NSAID-induced gastropathy, monitor any developing GI symptoms, and be willing to prescribe gastroprotective agents for the patient, in order to prevent serious adverse events. Also, the ultimate choice of therapy for a particular patient depends on several things including risk factors, the preferences of the patient and the physician, and $\operatorname{cost}^{10)}$.

\section{Conclusions}

The physician prescribing NSAIDS for arthritic knees should monitor any developing GI symptoms or the level of patient's own risk for gastropathy, and be willing to add gastroprotective agents for the patient in order to prevent serious adverse events.

\section{References}

1. Fries JF, Williams CA, Bloch DA, Michel BA. Nonsteroidal anti-inflammatory drug-associated gastropathy: incidence and risk factor models. Am J Med. 1991;91:213-22.

2. Lanas A, Ferrandez A. NSAID-induced gastrointestinal damage: current clinical management and recommendations for prevention. Chin J Dig Dis. 2006;7:127-33.

3. Zhang W, Moskowitz RW, Nuki G, Abramson S, Altman RD, Arden N, Bierma-Zeinstra S, Brandt KD, Croft P, Doherty M, Dougados M, Hochberg M, Hunter DJ, Kwoh K, Lohmander LS, Tugwell P. OARSI recommendations for the management of hip and knee osteoarthritis, Part II: OARSI evidence-based, expert consensus guidelines. Osteoarthritis Cartilage. 2008;16:137-62.

4. Richmond J, Hunter D, Irrgang J, Jones MH, Levy B, Marx R, Snyder-Mackler L, Watters WC 3rd, Haralson RH 3rd, Turkelson CM, Wies JL, Boyer KM, Anderson S, St Andre J, Sluka P, McGowan R; American Academy of Orthopaedic Surgeons. Treatment of osteoarthritis of the knee (nonarthroplasty). J Am Acad Orthop Surg. 2009;17:591600.

5. Korean Knee Society Subcommittee on Osteoarthritis Guidelines. Guidelines for the treatment of osteoarthritis of the knee. J Korean Knee Soc. 2010;22:69-74.

6. Lanas A, Tornero J, Zamorano JL. Assessment of gastrointestinal and cardiovascular risk in patients with osteoarthritis who require NSAIDs: the LOGICA study. Ann Rheum Dis. 2010;69:1453-8.

7. Huang JQ, Sridhar S, Hunt RH. Role of Helicobacter pylori infection and non-steroidal anti-inflammatory drugs in peptic-ulcer disease: a meta-analysis. Lancet. 2002;359:1422.

8. Graham DY, Chan FK. NSAIDs, risks, and gastroprotective strategies: current status and future. Gastroenterology. 2008;134:1240-6.

9. Bull SA, Conell C, Campen DH. Relationship of clinical factors to the use of Cox-2 selective NSAIDs within an arthritis population in a large HMO. J Manag Care Pharm. 2002;8:252-8.

10. Wolfe MM, Lichtenstein DR, Singh G. Gastrointestinal toxicity of nonsteroidal antiinflammatory drugs. N Engl J Med. 1999;340:1888-99.

11. Graham DY, Agrawal NM, Campbell DR, Haber MM, Collis C, Lukasik NL, Huang B; NSAID-Associated Gastric Ulcer Prevention Study Group. Ulcer prevention in long-term 
users of nonsteroidal anti-inflammatory drugs: results of a double-blind, randomized, multicenter, active- and placebocontrolled study of misoprostol vs lansoprazole. Arch Intern Med. 2002;162:169-75.

12. Chan FK, Ching JY, Hung LC, Wong VW, Leung VK, Kung NN, Hui AJ, Wu JC, Leung WK, Lee VW, Lee KK, Lee YT, Lau JY, To KF, Chan HL, Chung SC, Sung JJ. Clopidogrel versus aspirin and esomeprazole to prevent recurrent ulcer bleeding. N Engl J Med. 2005;352:238-44.

13. Silverstein FE, Graham DY, Senior JR, Davies HW, Struthers BJ, Bittman RM, Geis GS. Misoprostol reduces serious gastrointestinal complications in patients with rheumatoid arthritis receiving nonsteroidal anti-inflammatory drugs. A randomized, double-blind, placebo-controlled trial. Ann Intern Med. 1995;123:241-9.
14. Stockl K, Cyprien L, Chang EY. Gastrointestinal bleeding rates among managed care patients newly started on cox2 inhibitors or nonselective NSAIDs. J Manag Care Pharm. 2005;11:550-8.

15. Rich M, Scheiman JM. Nonsteroidal anti-inflammatory drug gastropathy at the new millennium: mechanisms and prevention. Semin Arthritis Rheum. 2000;30:167-79.

16. Inoue $N$, Nogawa $M$, Ito $S$, Tajima $K$, Kume $S$, Kyoi T. The enantiomers of etodolac, a racemic anti-inflammatory agent, play different roles in efficacy and gastrointestinal safety. Biol Pharm Bull. 2011;34:655-9.

17. Arakawa T, Kobayashi K, Yoshikawa T, Tarnawski A. Rebamipide: overview of its mechanisms of action and efficacy in mucosal protection and ulcer healing. Dig Dis Sci. 1998;43(9 Suppl):5S-13S. 